\title{
Change in Prevalence of Meeting the Aerobic Physical Activity Guideline Among US Adults, by States and Territories-Behavioral Risk Factor Surveillance System, 2011 and 2019
}

Emily N. Ussery, John D. Omura, Kelly McCain, and Kathleen B. Watson

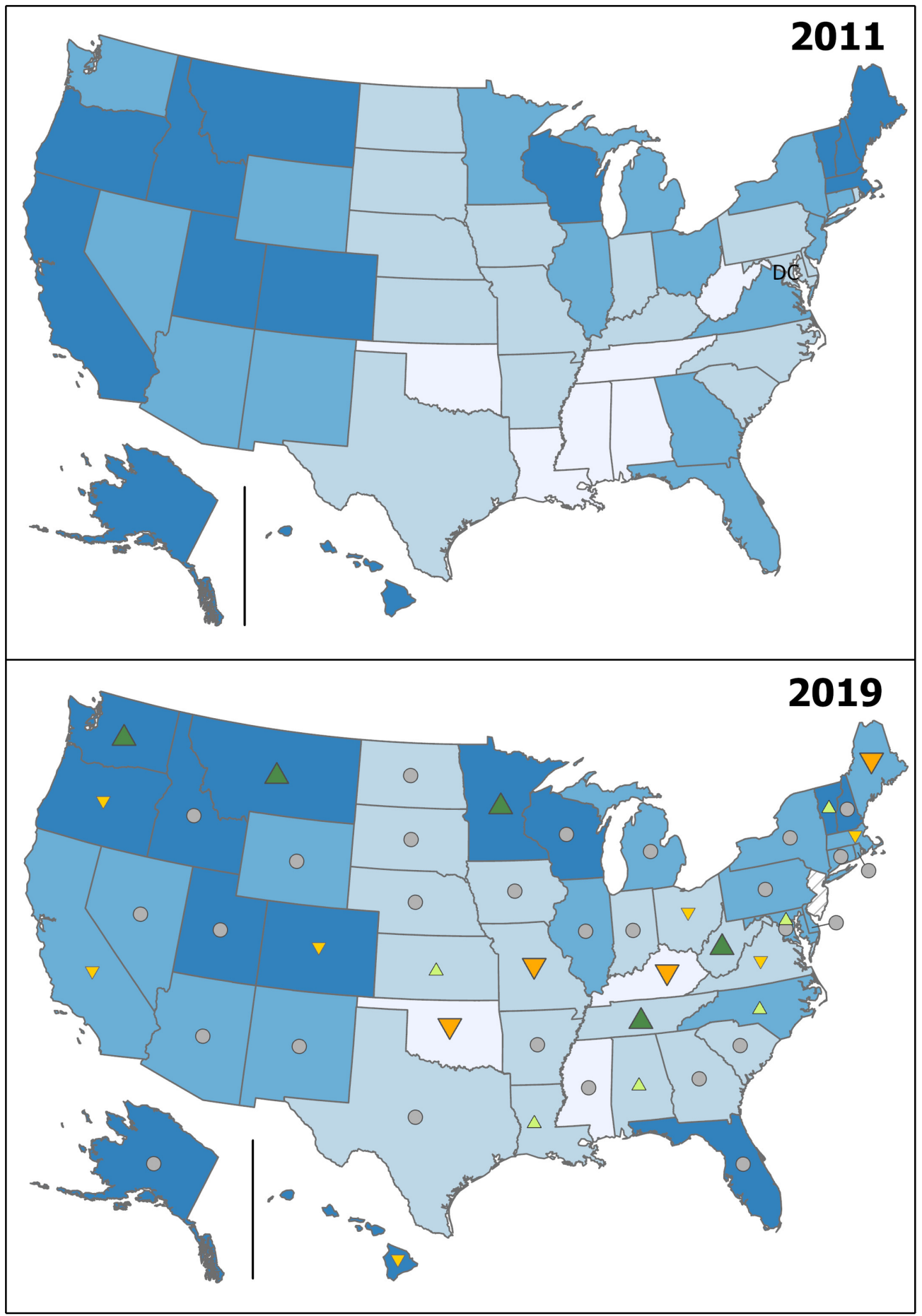

Prevalence of Meeting the Aerobic Physical Activity Guideline
$\square<45 \%$
$45 \%-<50 \%$
$50 \%-<55 \%$
$\geq 55 \%$
$\square$ Data unavailable

Absolute Change 2011 to $2019^{\text {III }}$

Significant change

$\checkmark>4$ percentage point decrease $>0-4$ percentage point decrease $>0-4$ percentage point increase

$\triangle>4$ percentage point increase

No significant change 
Overall, the percentage of US adults who met the aerobic physical activity guideline ${ }^{1},{ }^{\mathrm{I}}$ was $51.3 \%$ in 2011 and $51.3 \%$ in $2019(P=.876) .{ }^{\mathrm{II}}$ In 2011 , the prevalence of meeting the aerobic guideline was $<45 \%$ in 7 jurisdictions, $45 \%$ to $<50 \%$ in 16 jurisdictions, $50 \%$ to $<55 \%$ in 16 jurisdictions, and $\geq 55 \%$ in 14 jurisdictions. In 2019, the prevalence of meeting the aerobic guideline was $<45 \%$ in 4 jurisdictions, $45 \%$ to $<50 \%$ in 17 jurisdictions, $50 \%$ to $<55 \%$ in 16 jurisdictions, and $\geq 55 \%$ in 15 jurisdictions. Between 2011 and 2019 , the prevalence significantly decreased in 12 jurisdictions (CA, CO, HI, KY, ME, MA, MO, OH, OK, OR, VA, PR), significantly increased in 11 jurisdictions (AL, KS, LA, MD, MN, MT, NC, TN, VT, WA, WV), and did not significantly change in 29 jurisdictions. ${ }^{\text {III }}$ The absolute change in prevalence ranged from an 11.0 percentage point decrease in Kentucky to an 8.6 percentage point increase in Tennessee.

\section{Notes}

I Adult respondents (aged $\geq 18$ y) reported the frequency and duration of the 2 aerobic physical activities, outside of regular job duties, that they had spent the most time performing during the past month. The respondents were classified as meeting the aerobic physical activity guideline ${ }^{1}$ if they reported at least 150 minutes per week of moderate-intensity physical activity, at least 75 minutes per week of vigorous-intensity physical activity, or an equivalent combination of moderate- and vigorous-intensity physical activity (where vigorous-intensity minutes are multiplied by 2 ), totaling at least 150 minutes per week. The respondents whose physical activity level could not be categorized due to missing physical activity data were excluded (9.8\% of total). The estimates were weighted to account for the complex sampling design and nonresponse and to produce estimates representative of the adult population in each state/territory.

II Includes 50 states, District of Columbia, Puerto Rico, and Guam. Data were unavailable for New Jersey in 2019. To ensure a consistent comparison across years, New Jersey was excluded from national prevalence estimates in both years.

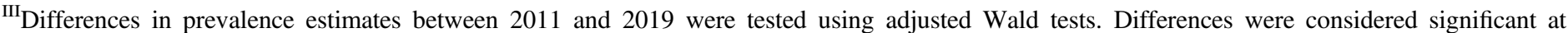
$P<.05$. States/territories with nonsignificant differences were categorized as "no significant change," and states/territories with significant differences were categorized according to the magnitude of the decrease or increase. Data from the 2011 BRFSS were used as the baseline for analysis due to changes in weighting and sampling methodology that were implemented in 2011.

Source: Behavioral Risk Factor Surveillance System, 2011 and 2019. https://www.cdc.gov/brfss/index.html. State-level estimates of additional physical activity measures from BRFSS with stratification by age, education, gender, income, and race/ethnicity can be found on the CDC's Data, Trends, and Maps interactive database: https://www.cdc.gov/nccdphp/dnpao/data-trends-maps/index.html

\section{Author Affiliations}

Ussery, Omura, and Watson are with the Division of Nutrition, Physical Activity, and Obesity; National Center for Chronic Disease Prevention and Health Promotion; Centers for Disease Control and Prevention; Atlanta, GA, USA. McCain is with the Geospatial Research, Analysis, and Services Program; Agency for Toxic Substances and Disease Registry; Centers of Disease Control and Prevention; Atlanta, GA, USA. Ussery (yzv4@cdc.gov) is corresponding author.

\section{Disclaimer}

The findings and conclusions in this report are those of the authors and do not necessarily represent the official position of the Centers for Disease Control and Prevention.

\section{Reference}

1. U.S. Department of Health and Human Services. Physical Activity Guidelines for Americans. 2nd ed. Washington, DC: U.S. Department of Health and Human Services; 2018. 